\title{
Filigrane
}

Écoutes psychanalytiques

\section{À propos de la neuropsychanalyse et de l'importance de penser le psychique}

\section{Ariane Bazan}

Volume 24, numéro 1, printemps 2015

Le devenir de la psychanalyse. Échos d'ici et d'ailleurs...

URI : https://id.erudit.org/iderudit/1033081ar

DOI : https://doi.org/10.7202/1033081ar

Aller au sommaire du numéro

\section{Éditeur(s)}

Revue Santé mentale au Québec

\section{ISSN}

1192-1412 (imprimé)

1911-4656 (numérique)

\section{Découvrir la revue}

\section{Citer cet article}

Bazan, A. (2015). À propos de la neuropsychanalyse et de l'importance de penser le psychique. Filigrane, 24(1), 27-41.

https://doi.org/10.7202/1033081ar

\section{Résumé de l'article}

Ce texte propose de situer ce nouveau domaine d'interface, la dite " neuropsychanalyse " dans la logique de l'histoire de la psychologie afin d'en articuler une épistémologie contre-intuitive et non-réductionniste. Le fil conducteur est l'idée que, de par l'histoire de la pensée, ce furent à chaque fois les progrès bouleversants de la biologie qui ont donné lieu au fondement, puis à l'institution du domaine de la psychologie. Or, nous nous trouvons à nouveau dans un moment de grand bouleversement par les progrès en neuro-imagerie. La visualisation cérébrale étant devenue totalisante, nous pensons qu'elle va acculer la psychologie à son heure de vérité. En effet, le mental, en cette époque du paradigme médical et neurobiologique, est soit mal pensé (sur un mode médical, induisant ainsi structurellement de la psychopathologie), soit n'est pas pensé du tout (réduit à un épiphénomène du biologique). Quand le dernier neurone sera finalement retourné, nous aurons enfin la certitude que l'âme ne s'y trouve pas ; il s'agira de fonder le psychique autrement, notamment à partir du sujet et non de la fonction. Le psychique pourra alors se révéler comme relatif à une perspective sur le corps, plutôt qu'à partir du corps. D'où la subversion du paradigme classique qui propose de "remonter du corps à l'âme ", comme dans ledit " dual aspect monism », soit l'épistémologie majoritairement en vigueur dans le domaine de la neuropsychanalyse. Nous nous démarquons de cette approche et souscrivons à un dualisme épistémologique, qui suggère que ce qui du sujet aidera à penser la physiologie, donnera consistance à une véritable science autonome du psychique. Plus précisément, nous proposons que les concepts de signifiant et de jouissance acquièrent une consistance du fait qu'ils peuvent offrir une grille de lecture éclairante de la physiologie du corps, voire même fonder une architecture de l'appareil psychique.
Ce document est protégé par la loi sur le droit d'auteur. L’utilisation des services d'Érudit (y compris la reproduction) est assujettie à sa politique d'utilisation que vous pouvez consulter en ligne.

https://apropos.erudit.org/fr/usagers/politique-dutilisation/ 


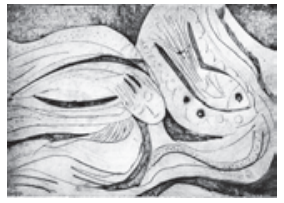

\title{
À propos de la neuropsychanalyse et de l'importance de penser le psychique
}

\author{
Ariane Bazan
}

Ce texte propose de situer ce nouveau domaine d'interface, la dite «neuropsychanalyse» dans la logique de l'histoire de la psychologie afin d'en articuler une épistémologie contre-intuitive et non-réductionniste. Le fil conducteur est l'idée que, de par l'histoire de la pensée, ce furent à chaque fois les progrès bouleversants de la biologie qui ont donné lieu au fondement, puis à l'institution du domaine de la psychologie. Or, nous nous trouvons à nouveau dans un moment de grand bouleversement par les progrès en neuro-imagerie. La visualisation cérébrale étant devenue totalisante, nous pensons qu'elle va acculer la psychologie à son heure de vérité. En effet, le mental, en cette époque du paradigme médical et neurobiologique, est soit mal pensé (sur un mode médical, induisant ainsi structurellement de la psychopathologie), soit n'est pas pensé du tout (réduit à un épiphénomène du biologique). Quand le dernier neurone sera finalement retourné, nous aurons enfin la certitude que l'âme ne s'y trouve pas; il s'agira de fonder le psychique autrement, notamment à partir du sujet et non de la fonction. Le psychique pourra alors se révéler comme relatif à une perspective sur le corps, plutôt qu'à partir du corps. D'où la subversion du paradigme classique qui propose de «remonter du corps à l'âme», comme dans ledit "dual aspect monism», soit l'épistémologie majoritairement en vigueur dans le domaine de la neuropsychanalyse. Nous nous démarquons de cette approche et souscrivons à un dualisme épistémologique, qui suggère que ce qui du sujet aidera à penser la physiologie, donnera consistance à une véritable science autonome du psychique. Plus précisément, nous proposons que les concepts de signifiant et de jouissance acquièrent une consistance du fait qu'ils peuvent offrir une grille de lecture éclairante de la physiologie du corps, voire même fonder une architecture de l'appareil psychique.

\section{L'avènement de la psychologie en réponse au dévoilement biologique}

A lors que depuis l'Antiquité, l'humain fut pensé sous le vocable de "philosophie», c'est au $\mathrm{XvI}^{\mathrm{e}}$ siècle que le mot psychologia apparaît pour la première fois (Mengal, 2000). Qu'est-ce qui a rendu nécessaire ce signifiant? En ce siècle de barbarie religieuse où les corps, souvent ouverts, jonchent la 
scène publique, les interdits officieux de l'église sur la dissection des corps humains sont dépassés par les faits. L'anatomie connait de grands progrès et, pour la première fois dans l'histoire de la pensée, le médecin Bruxellois André Vésale propose, avec ses planches anatomiques, une image des systèmes internes humains, et en particulier du système musculaire et des nerfs qui le parcourent. Ces images sont phénoménales et on peut supposer que leur effet est à la hauteur du bouleversement qu'engendre en ce moment l'imagerie cérébrale. En effet, elles découvrent que cette fabrique du corps se révèle comme une machinerie logiquement agencée et dont, par exemple, le mouvement se laisse comprendre mécaniquement par la beauté des emboîtements des muscles et des nerfs. Jusqu'alors, la «fysica» (les sciences naturelles) d'Aristote était la source principale des connaissances médicales dans les sphères d'influence juive, chrétienne et musulmane depuis quinze siècles. Elle proposait une prérogative de l'âme sur le corps: alors que le corps est une glaise qu'il faut réveiller à la vie par le souffle, c'est l'âme — l'anima qui précisément organise et insuffle son mouvement à ce corps. Cette doctrine médicale sous-tend l'art de guérir depuis si longtemps qu'elle semble construite pour durer toujours. Or, voilà qu'en ce Xvi ${ }^{\mathrm{e}}$ siècle, les dessins anatomiques la font trembler sur son socle: si ce n'est plus l'anima qui agite le corps, une anthropologie nouvelle, qui redéfinit cette âme s'impose. En 1540, le réformateur religieux allemand Philippe Melanchthon commente le De l'âme d'Aristote. Il enrichit le texte aristotélicien d'un long traité d'anatomie. Mais l'addition simple ne suffit pas, les images anatomiques du corps ont fondamentalement brouillé les anciennes cartes: puisque le corps semble en mesure de reprendre une série de fonctions, auparavant réservées à l'âme (comme le mouvement mais aussi la chaleur du sang), il faut redéfinir cette âme. À la position d'Aristote — soit que tout être vivant, qu'il soit végétal, animal ou humain, possède à des degrés divers une âme qui organise le corps - Melanchthon oppose alors une anthropologie dualiste qui divise l'homme en un corps mouvant étendu dans l'espace et une âme pensante. L'homo duplex de Melanchton, implique que désormais, l'anthropologia soit divisée en deux parties: l'anatomia, l'étude du corps, et pour la première fois la psychologia, l'étude de l'âme. L'utilisation du terme par Melanchthon est sa première utilisation intentionnelle et elle fonde un nouveau champ de la connaissance. Dans son sillage, le réformateur hollandais Rudolph Snellius (1594, p. 26-27) précise alors les propriétés essentielles qui distinguent corps et âme: 
L'âme raisonnable de l'homme est la pensée qui, conjuguée au corps, parachève l'homme. [...] Les choses physiques plus proches des corps naturels qui se meuvent naturellement, possèdent une étendue et à cause de cela occupent un lieu.

La faculté de l'âme rationnelle est la pensée ou volonté. La pensée est la faculté de l'âme de discourir et de penser à propos des choses qui sont et ne sont pas. (Mengal, 2000/2001, p. 10)

De façon judicieuse, la pensée est définie comme la capacité de se représenter les choses sans qu'elles ne doivent être au moment même présentes, comme en témoigne l'imagination. Cette âme, nouvellement menacée par les progrès de l'anatomie, se voit donc repêchée par l'attribution de nouvelles exclusives, notamment la pensée (l'imagination) et la volonté. René Descartes (1648) connaissait les progrès fulgurants de son époque en matière d'anatomie; lui-même procédait à la dissection de corps animaux et humains. Il en vient à la conclusion que le corps est un appareil complexe capable de se mouvoir sans l'aide de l'âme:

L'âme ne peut exciter aucun mouvement dans le corps, si ce n'est que tous les organes corporels, qui sont requis à ce mouvement, soient bien disposés; mais que, tout au contraire, lorsque le corps a tous les organes disposés à quelque mouvement, il n'y a pas besoin de l'âme pour les produire. (Descartes, 1648/1964-74, p. 225)

Notons que c'est en tant que philosophe que Descartes propose sa vision dualiste, qui correspond aux points de vue réformistes. À aucun moment Descartes n'emploie le mot «psychologie»; ce sont les médecinsréformateurs Melanchthon, Goclenius et Snellius qui introduisent le terme (Mengal, 2000).

Ce nom de «psychologie» émerge donc de la nécessité de penser l'âme en réponse au menaçant dévoilement de l'humain par la biologie. Ce paradoxe se répète à la moitié du XIX ${ }^{e}$ siècle, quand la psychologie, jusque là largement considérée comme une branche de la philosophie, s'émancipe en un domaine scientifique autonome. Et, à nouveau, c'est à l'aune des grands progrès de la biologie que ce remaniement a lieu. En effet, le xix ${ }^{e}$ siècle se caractérise par nombre de découvertes fondamentales pour la physiologie, incluant la neurophysiologie. Charles Bell et François Magendie découvrent indépendamment la distinction entre nerfs sensoriels et moteurs au niveau 
de la moelle épinière; Emil du Bois-Reymond met en carte la nature électrique de la contraction musculaire; Pierre Paul Broca et Carl Wernicke identifient les aires du cerveau qui interviennent dans différents aspects du langage et Gustav Fritsch, Eduard Hitzig et David Ferrier localisent les aires sensorielles et motrices du cerveau (voir, p. ex. Brennan, 1998). Le physiologiste expérimental Hermann von Helmholtz étudie un large éventail de phénomènes, tel la nature du son et de la couleur, ainsi que leur perception (Warren et Warren, 1968). Dans les années 1860, Helmholtz engage à Heidelberg un jeune assistant médecin, Wilhelm Wundt. Wundt utilise l'appareillage du laboratoire physiologique pour étudier expérimentalement les principes de la perception sensorielle. Il applique la méthode des mesures de temps de réaction, une mesure propre à la recherche en psychologie. En 1874, il rédige le premier manuel de psychologie Principes de psychologie physiologique ${ }^{1}$ et en 1879 , il établit le premier laboratoire qui sera spécifiquement dédié à la recherche originale en psychologie expérimentale. La psychologie comme discipline scientifique autonome voit le jour.

En d'autres termes, c'est la confrontation avec la complexité époustouflante du corps, ensuite du cerveau, qui au XVI ${ }^{\mathrm{e}}$ et au XIX ${ }^{\mathrm{e}}$ siècles, a contraint la pensée à fonder le domaine de la psychologie. Ce qui semble se passer à chaque fois est la reconnaissance que ce qui fut jadis attribué à l'âme est en fait réalisé par le corps. Il est très paradoxal que précisément cette reconnaissance mène alors à la promotion de la psychologie comme domaine autonome. Au xvi ${ }^{\mathrm{e}}$ siècle la mesure est prise de ce que l'anatomie peut expliquer en elle-même le mouvement du corps et en contrecoup, ceci aboutit à la fondation d'une doctrine avec un nouveau nom, la psychologie, distincte de l'anatomie et réservée aux qualités qui sont spécifiques à l'âme, telles la pensée, la parole et la volonté. Au Xix ${ }^{e}$ siècle la mesure est prise de ce que la neurophysiologie peut expliquer la perception et le langage et ceci, à son tour, institue définitivement la psychologie comme discipline scientifique, distincte de la philosophie.

Paradoxalement, elle l'institue également comme distincte de la physiologie, bien qu'elle y soit solidement ancrée: en effet, certains physiologistes qui sont des premiers "psychologues» proposeront qu'elle se caractérise non pas par des fonctions propres, mais bien par des lois propres. En 1867, Wundt, par exemple, rejette l'approche naïvement matérialiste et défend l'idée de l'autonomie du psychique: les lois qui gouvernent le mental sont fondamentalement différentes de celles qui gouvernent la nature matérielle. Le philosophe John Stuart Mill (1882 [1843], 590, 593, 490) défend également 
l'autonomie d'un niveau psychologique d'analyse et rejette l'hypothèse des phénomènes mentaux comme «générés par l'intervention de mécanismes matériels». L'étude des phénomènes mentaux nécessite des lois invariables qui sont à distinguer non seulement de la métaphysique, mais également des approches physicalistes de l'esprit ou de la psychologie biologisée. La psychologie doit décrire «les uniformités de succession, les lois, qu’elles soient ultimes ou dérivées, grâce auxquelles un état mental succède à un autre, est causé par un autre, ou, du moins, qui sont cause du fait qu'un état mental suit un autre». Le physiologue Helmholtz (1896 [1877], 187), par ailleurs, propose que «la mémoire, l'expérience et l'habitude» soient des «faits, dont les lois sont à chercher, et qui ne peuvent être résolus [explained away] parce qu'ils ne peuvent [...] être attribués aux lois connues de l'excitation nerveuse». Il est donc à noter qu'en cette fin de $\mathrm{XIX}^{\mathrm{e}}$ siècle quelques-uns des tenants majeurs de la psychologie de l'époque cherchent à fonder la psychologie dans des lois et des régularités propres au domaine.

\section{Les drames du mal-penser et du non-penser de la psychologie}

$\mathrm{Au} \mathrm{xx} \mathrm{x}^{\mathrm{e}}$ siècle, Sigmund Freud, avec la psychanalyse, propose une science de l'âme dont les principes organisateurs s'affranchissent radicalement tant de la physiologie que de la philosophie. Freud, qui a une formation de neurologue, et qui s'inscrit dans la lignée de l'école physicaliste de Berlin, est fidèle aux enseignements de ses maîtres Ernst Brucke et, surtout, Hermann von Helmholtz. Il réussit ce tour de force qu'est la métapsychologie psychanalytique; à l'instar de son autre maître — du côté de la philosophie Mill, il propose ainsi une véritable science autonome de l'âme dont les lois s'affranchissent du biologique tout en restant fidèle à l'approche transcendantale des physiologistes Fichte et von Helmholtz. Muni d'une expertise en neurophysiologie, Freud en vient à supposer le fonctionnement physiologique de l'appareil psychique afin de rendre compte de ce qu'il observe dans la clinique. Ceci aboutit alors en 1895 à l'Esquisse d'une psychologie scientifique où il introduit, par exemple, les concepts de processus primaires et secondaires pour décrire le mental; les processus primaires semblent alors directement inspirés des lois proposées par Mill, à savoir les lois de l'association par contiguïté et ressemblance.

Or, l'avènement des psychotropes dans les années 1950, conjugué aux avancées époustouflantes en imagerie cérébrale des trente dernières années, ont de nouveau bouleversé le domaine de la psychologie et ont, en particulier, réinsufflé vie au paradigme partialisant dit « résolutif-recompositif » des 
débuts. En effet, le modèle fondateur de la psychologia est emprunté à l'anatomie: puisque la science procède par découpage, par la mise en évidence du plus simple, et qu'elle recompose à partir du plus simple le plus complexe, la psychologie a pour but de décrire ses facultés comme l'anatomie découpe le corps en ses parties. Le philosophe empiriste David Hume (1938 [1740], $6 ; 1969$ [1739/40],311), par exemple, décrit son projet en termes d'une anatomie de la nature humaine, c'est-à-dire qu'il propose de décomposer les phénomènes mentaux en éléments plus primitifs (impressions et idées) et de reconstruire leur histoire formative à l'aide d'un nombre minimal de lois mentales. Wundt (1882: 399), par ailleurs, propose que les objectifs de la psychologie scientifique soient «la complète décomposition [Zergliederung] des phénomènes conscients en leurs éléments ». Les avancées récentes des neurosciences permettent de totaliser cette ambition: en effet, rien de ce qu'on aurait voulu arroger à l'intime de l'âme, rien de la passion, de l'amour, de l'amitié, des aspirations ou même de la foi, de la morale, du désir ou de la jouissance orgastique, rien n'échappe à la visualisation. Quoi de plus étonnant que d'en déduire que le psychique ne serait rien d'autre qu'une sorte de phénoménologie ou d'expression directe de processus cérébraux? La conséquence paradoxale de ces avancées semble alors être, logiquement, la conception de la psyché comme une phénoménologie du corps, régie par les lois biologiques et médicales d'une part, et l'impossibilité de penser la spécificité du psychique d'autre part.

Nous proposons maintenant que tant ce «mal-penser» que ce «nonpenser» soient structurellement voués à la faillite et au drame. Pour ce qui est du «mal-penser», si la psyché est régie par les lois biologiques, il s'ensuit qu'il s'agit de traiter l'âme selon les mêmes principes que ceux en vigueur pour traiter le corps, c'est-à-dire, les principes médicaux. Nous avons proposé ailleurs (Bazan, 2013) que ces principes médicaux, bien qu'ayant mené à de précieuses et spectaculaires avancées dans la médecine du corps, sont au contraire contreproductifs quand ils s'appliquent à la santé mentale. Citons brièvement trois de ces principes. Premièrement, précisément le diagnostic par découpage du problème de sa chaîne logique — avec parallèlement l'intervention par la spécialisation clinique pour les différentes composantes fait perdre son sens au symptôme. Ne reste au clinicien «spécialiste» qu'une approche essentialiste, qui explique le problème par une essence, une nature ou une prédisposition (Hyman, 2010). Cette essence est alors cristallisée par un nom, le nom d'un diagnostic. Or, ceci fixe le problème pour le sujet en l'y identifiant, sans proposer de réelles pistes de prise en charge (voir 
p. ex. Casper, 2008). Moncrieff et d'autres (1995; Moncrieff et Kirsch, 2005; Moncrieff et Timimi, 2010), par exemple, montrent que les labels diagnostiques sont moins utiles qu'une simple description des problèmes pour la prédiction de la réponse au traitement. Qui plus est, cette approche essentialiste entraîne, à son tour, l'aspiration à déterminer le profil distinctif de ces sujets avec le «même» problème et de nouveaux «troubles de la personnalité» voient le jour. Toutefois, cette approche a des effets propres (Hacking, 1985, 102-103): en effet, les sujets, mis à mal par leur détresse et leur désarroi, ou ceux de leurs proches, cherchent à saisir ces maux, et se trouvent (temporairement) soulagés à pouvoir y coller une étiquette. Mais même la British Psychological Society indique que «les clients, hélas, trouvent le plus souvent que le diagnostic n'offre qu'une fausse promesse de tels avantages [de reconnaissance]» et que "les diagnostics sont décidemment inutiles comparés à d'autres alternatifs ${ }^{2} »$. La proposition d'un nouveau diagnostic augmente la probabilité d'émergence de ce diagnostic dans la population, et peut ainsi contribuer à créer l'épidémie. Par exemple, Ethan Watters (2010) rapporte qu'à Hong Kong, la première description de l'anorexie mentale dans les médias en novembre 1994 a précédé l'apparition explosive de cette pathologie qui y était auparavant pratiquement inconnue (voir aussi p. ex., Kutchins, 1997). En ce qui concerne le deuxième principe médical, le dépistage et la prévention, selon la même logique, dans le domaine de la santé mentale, ces campagnes, ciblées sur les composantes découpées, ont un effet inverse à celui espéré: le dépistage actif induit un appel d'air à l'identification aux troubles et contribue ainsi à provoquer des vagues d'épidémies psychopathologiques. De plus, l'élément constitutif de l'offre clinique de l'approche médicale est souvent le médicament psychotrope, même si les modalités de l'effet thérapeutique de la plupart des psychotropes ne sont pas bien comprises. Qui plus est, leurs effets ciblent surtout les symptômes, privilégiant parfois la diminution de ceux-ci au soutien du fonctionnement psychosocial (Wunderinck et al., 2007). En outre, les bénéfices à long terme des psychotropes sont parfois mis en doute; par exemple, une étude récente démontre qu'après 20 ans, un groupe de schizophrènes n'ayant pas reçu de médicaments antipsychotiques a significativement moins d'activités psychotiques qu'un groupe de patients neuroleptisés (Harrow et al., 2014). La discussion de ces différents aspects demanderait un débat plus étayé ${ }^{3}$, mais nous nous permettons, pour l'heure, d'en retirer l'idée que l'application des principes médicaux dans le champ de la santé mentale induirait de la psychopathologie plutôt qu'elle ne la soignerait. 
Par ailleurs, le choix de l'approche médicale doit être relié à une véritable impossibilité de penser le psychique tant dans les arènes scientifiques, que, par retour d'effet, dans le "grand public». Par exemple, le neurologue Robert Burton (2013) dit dans son livre A Skeptic's Guide to the Mind, que même après 2500 années de contemplation et de recherche, nous n'avons toujours «aucune idée de ce qu'est le mental». Le domaine de la psychologie ne semble pas réellement offrir de réponse à la question: si tant est que les fonctions et les instances psychologiques peuvent être mises en correspondance avec des structures précises et déterminées du cerveau, en quoi la psychologie est-elle différente de la neurophysiologie? Que veut dire psychologie au-delà du cerveau? Loin d'être anodin, nous proposons que cet impossible peut mener «à la perte» pour les sujets. En effet, il semble aujourd'hui le plus souvent impossible de penser la souffrance autrement qu'en termes de paramètres biologiques ou sociologiques: si ce ne sont pas les gènes, les hormones, les neurones, alors ça doit être l'éducation, la famille, le contexte, la société, et vice versa. Il n'y a donc de sujet que du corps ou du social, c'està-dire, qu'il n'y a de sujet que victime ou objet. Il s'ensuit que ces « victimes » réclament des compensations, des droits et qu'elles guettent les coupables possibles, qui sont toujours externes et «autres». Puisqu'il n'y a pas de sujet, il n'y a pas d'éthique du sujet, ni de sujet mobilisable dans son rôle organisateur du malheur qui lui arrive. En conséquence, tel Sysiphe, incapable de se rendre compte de sa propre implication, ces sujets se retrouvent condamnés à répéter indéfiniment.

\section{Un troisième temps pour la psychologie}

Pourtant, nous avons des raisons de nous réjouir: en effet, de «la source de tous les maux» peut venir le salut. En effet, les conséquences paradoxales des techniques de pointe en neuro-imagerie mettent les neurosciences dans un certain embarras. Maintenant que nous voyons de mieux en mieux, nous voyons "tout», et ce tout est vertigineux: dans le cerveau tout est interconnecté. Néanmoins, depuis que nous voyons tout, nous pouvons enfin prendre la mesure de ce qu'en vérité, nous ne voyons rien: le corps ne se donne pas à lire, il ne se présente pas avec une grille de lecture attachée. Il n'existe pas de vérité sur la nature humaine qui se donnerait à lire par le neurone. Tant que nous n'aurons pas été jusqu'au bout de la voie offerte par la neuro-imagerie, l'illusion aura de quoi se nourrir au moindre doute: «mais quand nous aurons des techniques plus performantes, à ce moment là...». Or, quand l'excès de données aura finalement 
effacé tout relief permettant une lecture — de la même façon qu'à trop bien voir la granulation de la photo on en perd l'image — alors s'articulera de façon la plus pressante et la plus précise la nécessité d'une grille de lecture à partir d'un autre niveau.

Nous proposons donc que la neuro-imagerie actuelle sonne un troisième temps pour la psychologie et que, du fait même de son dévoilement totalitaire, elle accule structurellement cette psychologie à son heure de vérité (voir aussi Bazan, 2011) : soit elle est exhaustivement remplacée par les neurosciences et elle disparaît en tant que telle, soit le domaine se fonde de façon radicalement différente, et peut-être pour la première fois de façon véritablement autonome. À cet endroit, nous proposons (en accord avec Mill, Wundt et von Helmoltz) que ce ne soient pas tant les modules, les composantes ou les capacités qui distinguent le psychologique du biologique, mais bien l'échelle organisationnelle par laquelle elle les considère — de la même façon d'ailleurs que la biologie ne differe pas de la chimie par ses composantes mais qu'elle les considère à une autre échelle (voir aussi Bazan, 2011). Plus précisément, nous proposons que la psychologie s'adresse spécifiquement au niveau du sujet plutôt qu'à celui de la fonction. Il s'agirait maintenant d'arrêter une définition de ce sujet (comme on a pu le faire précédemment pour la fonction), mais la logique du raisonnement proposé n'oblige pas à se restreindre à une seule définition, du moment qu'il s'agisse d'un niveau qui permette une perspective sur le corps plutôt qu'une perspective à partir $d u$ corps. Nous proposons que ce sujet-là fonde le domaine du psychique en sa spécificité et que, paradoxalement, la révolution de l'imagerie cérébrale détient en son sein la perspective de ce fondement.

\section{Psychanalyse et neurosciences}

Une méthodologie spécifique à la psychologie et qui livre le matériel pour la proposition de concepts cohérents au niveau du sujet est alors l'écoute clinique. Parmi les cadres qui s'inscrivent dans cette épistémologie du sujet, il y a la psychanalyse. Si la psychanalyse a pu proposer des lois propres au fonctionnement mental (p. ex. les processus primaires et secondaires, le signifiant, la jouissance), son histoire montre que cette pensée est passée, pour ce qui en est de Freud, par le biologique: c'est à partir des limites du biologique que la métapsychologie se fonde. C'est par ce que le biologique héberge d'impossible à penser qu'il ouvre l'espace, qu'il désigne le lieu d'où peut se penser le psychique. En d'autres termes et paradoxalement, la biologie est vitale pour la psychanalyse. 
Biologie et psychanalyse, neurosciences et psychanalyse, s'entrecroisent en particulier dans ce domaine dit de la «neuropsychanalyse». La neuropsychanalyse est parfois - et parfois, à juste titre — considérée comme un barbarisme, tant par son nom que par sa chose. La ligne épistémologique la plus suivie est celle de Solms et Turnbull (2002), notamment le dual aspect monism, c'est-à-dire l'idée qu'il n'y a qu'un objet, qui peut être caractérisé de façon objective ou neuroscientifique — le cerveau — soit de façon subjective ou clinique - l'appareil mental. En fin de compte, il y aurait une correspondance plus ou moins linéaire entre les paramètres cérébraux et psychiques: chaque phénomène qui trouve une cohérence au niveau du cerveau correspond à un phénomène qui trouve une cohérence au niveau psychique. La recherche opère selon le paradigme classique: des observations neurophysiologiques sont mises en correspondance avec des caractéristiques comportementales ou de personnalité. De ce fait, même dans une perspective psychanalytique, l'on aboutit à une sorte de mise en miroir: l'âme reflète le cerveau. Ce paradigme implique que la connaissance du cerveau puisse (directement) contribuer à la clinique psychanalytique.

Nous rejetons cette approche: nous proposons, au contraire, qu'il n'y a pas d'objet en soi qui soit connaissable, c'est-à-dire, en tant qu'objet (inerte et construit) indépendamment de sa perception. Nous nous inscrivons dans une approche transcendantale selon Kant, qui propose que la façon dont ce qui de la nature ${ }^{4}$ fera objet, est empreinte de la façon dont cette nature est saisie, c'est-à-dire que l'objet se construit dans la négociation entre un sujet saisissant et une nature qui résiste (Van de Vijver et Demarest, 2013). L'idée n'est pas que le sujet puisse déterminer ou exhaustivement construire l'objet, mais l'objet est néanmoins marqué de la question qui l'a fait apparaître. Selon cette approche, la biologie construit un objet «cerveau» d'une part et la clinique un objet «appareil mental» de l'autre, mais il n’y a pas nécessairement de correspondance linéaire entre les deux. Ce qui fait cohérence dans le cerveau ne peut pas être mis en correspondance point par point avec ce qui fait cohérence dans l'appareil mental; l'âme n'est pas le miroir du substrat neuronal. Il s'agit donc d'une forme de dualisme, même si ce dualisme n'est pas ontologique. On pourrait dire qu'il s'agit d'un dualisme épistémologique, c'est-à-dire, d'une approche qui refuse d'expliquer les états mentaux en termes d'états du corps.

Cette épistémologie s'inscrit dans une considération plus générale de l'organisation de la matière et du vivant. L'idée que, bien que la biologie émerge de la chimie, ces deux sciences constituent néanmoins des niveaux 
d'organisation autonomes de la matière, qui ne sont pas dans un rapport réciproque d'exacte correspondance et qui bénéficient de leur propre appareil conceptuel, d'analyse et d'intervention, ne fait généralement pas grand débat. La psychologie ne se rapporte pas différemment à la biologie: bien qu'il puisse être important pour certains aspects de «retourner» au substrat biologique, il n'en serait pas moins absurde de tenter de remplacer «en fin de compte» des phénomènes psychiques par des dynamiques biologiques. Il est évident que ceci n'implique pas que la psyché apparaîtrait tel un génie d'une lampe. Le psychique se constitue dans le champ de tension entre un substrat biologique qui pousse et un niveau social qui tire, c'est-à-dire à partir d'une pression pulsionelle et en réponse à un autre appelant. De cette ontologie, il résulte que l'appareil psychique est marqué tant de la biologie que de l'autre. La dimension «neuropsychanalytique» dans notre approche tente alors de caractériser les points d'attache ou de nouage entre le biologique et le psychique, c'est-à-dire d'articuler comment un même phénomène au cœur d'un tel nœud peut s'épeler de façon tant biologique que psychique. Dans tous les cas, il s'agit de phénomènes qui rendent compte au niveau psychique de la condition humaine à vivre dans un corps humain. De tels nœuds témoignent d'une correspondance directe entre biologique et psychique. Il y a néanmoins une différence essentielle avec le modèle moniste: la biologie impliquée dans ces nœuds ne dicte pas en soi l'organisation du psychique, n'a pas un rôle organisateur pour le mental, mais fonctionne comme contrainte, comme point d'achoppement qui limite en même temps qu'elle rend possible la constitution mentale (Van de Vijver, 2010).

Notre approche des neurosciences est donc subvertie: ses progrès n'aboutiront pas à un nouveau savoir sur la clinique du sujet, mais inversement, la clinique peut mener à proposer des concepts qui permettent de lire la physiologie, de débroussailler l'excès de données au niveau physiologique - en d'autres termes, à un savoir sur le corps. Le propos se renverse donc: c'est, en effet, ce qui du sujet ${ }^{5}$ pourra aider à penser la physiologie qui pourra faire étoffe, qui pourra donner consistance à un véritable appareil psychique avec une architecture propre. Ce n'est pas le substrat physiologique qui démontre le concept clinique, mais c'est le concept clinique qui, puisqu'il s'avère capable de rassembler un nombre d'observations physiologiques disparates, acquiert une pertinence heuristique.

Nous avons précédemment proposé deux possibles nœuds entre les niveaux biologique et psychique: le signifiant (Bazan, 2007) et la jouissance (Bazan et Detandt, 2013). Il s'agit en même temps de deux dimensions 
cliniques de toute relation transférentielle, soit l'irrationnel et le transgressif. Voici nos propositions. Le signifiant est le phénomène qui rend compte au niveau psychique de la condition spécifiquement humaine du langage. À la différence de toute forme animale de langage, les phonèmes du langage humain sont au plus haut point dépendants des phonèmes environnants pour leur interprétation, c'est-à-dire du contexte. Cette dépendance éminemment contextuelle de leur interprétation impose une exigence colossale à la tendance naturelle du fonctionnement du cerveau, c'est-à-dire l'inhibition de l'élan systématique et spontané de l'interprétation directe dictée par le stimulus (le stimulus phonologique en particulier). Les phénomènes de signifiant «trahissent» que nous n'y arrivons pas tout le temps ou pas de façon exhaustive: dans la décompensation psychotique par exemple, le sujet est assailli par la polysémie du langage qui, par son effet associatif tout azimut fait l'effet d'une bombe. En dehors de la psychose, il y a également des symptômes structurés par le signifiant (des phobies, des rituels, des préférences, des dégoûts) qui trahissent que le langage ne s'interprète pas exclusivement contextuellement mais qu'il peut, en sa qualité d'objet à charge affective, fixer une tendance du sujet. Au niveau biologique, le signifiant est un fragment phonémique alors qu'au niveau psychique, il est une tendance propre au sujet et au niveau social, une dimension irréductible de la déraison (et de la folie) humaine.

La jouissance est le phénomène qui rend compte au niveau psychique de la condition du corps pulsionnel. Les premières sources pulsionnelles se situent dans le corps invertébré, le «sac» de viscères, dont le système respiratoire, la circulation sanguine, le système digestif, le système d'excrétion et le système de reproduction. Des besoins ou des alarmes du corps interne sont traduits en un excès d'excitation qui incite le corps externe, le corps vertébré — le squelette et les muscles squelettiques - à agir. Chez de nombreuses espèces animales ces deux corps sont raccordés de façon «naturelle»: un poulain nouveau-né qui a faim, par exemple, peut se mettre sur ses pattes et s'avancer vers le téton de la jument. Dans d'autres espèces animales, et en particulier chez l'homme, il y a une vraie béance entre corps interne et corps externe. Quand il y a alarme au niveau du corps interne, l'humain est incité à agir (avec son corps externe), mais cette action n'est pas dirigée naturellement vers une action qui serait adéquate par rapport à l'alarme: le nouveauné qui a faim, par exemple, se met à crier et à agir, des actions qui en soi ne calment pas la faim mais qui permettent une décharge non-dirigée. Quand par accident, ou grâce à l'aide d'un congénère, une action adéquate est 
trouvée (par exemple, la mère met l'enfant à son sein), alors cette séquence d'actions (p. ex. la succion) est récompensée biologiquement et inscrite dans l'histoire du corps; ceci se ferait par le biais du système mésolimbique (voir Bazan et Detandt, 2013). Cette inscription a pour conséquence structurelle, cependant, que l'action et son résultat sont découplés puisqu'elle incite à répéter l'action en tant que séquence motrice en soi, et indépendamment de son résultat. Dans la nature, il est rare qu'une action mène à des résultats radicalement différents d'avec le résultat inaugural. Dans la culture, cependant, les changements de contextes sont pléthores, et une action qui fut à l'origine adéquate (par exemple, l'enfant qui se tient immobile en réponse à une mère surmenée et irritable) peut être à d'autres moments non adéquate ou même dommageable (par exemple, l'adulte qui se tient à carreau dans des interactions professionnelles ou sociales). La jouissance «trahit» l'inscription de séquences d'actions indépendamment de leur résultat. Au niveau biologique, la jouissance serait l'inscription mésolimbique de l'action " adéquate ${ }^{6}$ », au niveau psychique il s'agit d'une compulsion à répéter singulière au sujet, et au niveau social, d'une dimension irréductible de la transgression (puisque le sujet est attaché à l'agir en soi).

Le potentiel heuristique du signifiant et de la jouissance serait à même de fonder une architecture psychique. Bien que dans les deux cas, il soit possible de proposer un substrat biologique, ces deux concepts échappent néanmoins à la logique du miroir. Pour chacun, il existe, au-delà du nouage avec le biologique, un déploiement théorique particulièrement articulé et basé sur la clinique, qui s'émancipe radicalement du biologique. Ce déploiement théorique peut ainsi donner un poids conceptuel à la notion d'appareil mental, alors que la pertinence de son articulation biologique atteste du fait que l'âme se concrétise à travers sa condition corporelle et, en particulier, des contraintes spécifiques que ce corps lui impose.

Ariane Bazan

Ariane.Bazan@ulb.ac.be

\section{Notes}

1. Grundzüge der Physiologische Psychologie.

2. The British Psychological Society Response to the American Psychiatric Association: DSM-5 Development, June 2011.

3. Pour une discussion plus étayée, voir p. ex. Gonon, 2011.

4. De la matière - soit encore, du Réel dans une perspective Lacanienne.

5. Ou du psychique, ou de la maladie mentale. 
6. Mais aussi de toute action en cas de trauma, puisque dans ce cas, toute action est mieux que la non-action, indépendamment de son résultat; en cas de trauma, toute décharge ou toute mise en forme déchargeable, du surplus d'excitation est, d'une certaine façon, «adéquate» (Bazan et Detandt, à paraître).

\section{Références}

Bazan, A. (2007). Des fantômes dans la voix. Une hypothèse neuropsychanalytique sur la structure de l'inconscient. Montréal: Editions Liber.

Bazan, A. (2011). The grand challenge for psychoanalysis — and neuropsychoanalysis: taking on the game. Frontiers in Psychology, 2, 220. doi: 10.3389/fpsyg.2011.00220.

Bazan, A. (2013). Een ziel laat zich niet als een lijf versnijden. Over de gevolgen van het medisch model voor de mentale gezondheidszorg. Dans I. Devisch (dir.), Ziek van gezondheid. Voor elk probleem een pil? (p. 121-148). Anvers, Amsterdam: De Bezige Bij.

Bazan, A. et Detandt, S. (2013). On the physiology of jouissance: interpreting the mesolimbic dopaminergic reward functions from a psychoanalytic perspective. Frontiers in Human Neuroscience, 7, 709. doi: 10.3389/fnhum.2013.00709

Bazan, A. et Detandt, S. (à paraître). Trauma and jouissance, a neuropsychoanalytic perspective. Journal of the Centre for Freudian Analysis and Research (JCFAR).

Brennan, J.F. (1998). History and Systems of Psychology. London: Prentice-Hall International. Burton, R. (2013). A Skeptic's Guide to the Mind: What Neuroscience Can and Cannot Tell Us About Ourselves. New York: St. Martin's Press.

Casper, M.-C. (2008). Le diagnostic comme effet de nomination. Un exemple la dyslexie. L'évolution psychiatrique, 73, 485-495.

Descartes, R. (1648) La description du corps humain. Dans C. Adam et P. Tannery (dir.), Euvres de Descartes (1897-1913), Vol. XI. Paris: L. Cerf; nouvelle édition complétée, Vrin-CNRS, 1964-1974.

Gonon, F. (2011). La psychiatrie biologique: une bulle spéculative? Esprit, novembre, 54-73.

Hacking, I. (1985). Making Up People. Dans T.L. Heller, M. Sosna et D.E. Wellerby (dir). Reconstructing Individualism (p. 222-236). Stanford: Stanford University Press.

Harrow, M., Jobe, T. et Faiull, R. (2014). Does treatment of schizophrenia with antipsychotic medications eliminate or reduce psychosis? A 20-year multi-follow-up study. Psychological Medicine. March, 2014. doi: 10.1017/S0033291714000610.

Hatfield, G. (1990). The Natural and the Normative. Theories of Spatial Perception from Kant to Helmholtz. London: MIT Press.

Helmholtz, H. (1896). Das Denken in der Medicin. In Helmholtz, Vorträge und Reden. 2,165190. Braunschweig: Holzstiche, 1877.

Hobson, J.A. et McCarley, R.W. (1977). The brain as a dream state generator: an activationsynthesis hypothesis of the dream process. American Journal of Psychiatry, 134, 1335-1348.

Hume, D. (1938). An Abstract of a Treatise of Human Nature. Cambridge: Cambridge University Press, 1740.

Hume, D. (1969). A Treatise of Human Nature. London: Penguin Books, 1739-40.

Hyman, SE. (2010). The diagnosis of mental disorders: the problem of reification. Annual Review of Clinical Psychology, 6, 155-179.

Jeannerod, M. (1994). The representing brain: neural correlates of motor intention and imagery. Behavioral and Brain Sciences, 17, 187-245.

Jeannerod, M. (2002). La Nature de l'esprit. Paris: Odile Jacob.

Kutchins, H. et Kirk, S. (1997). Making Us Crazy: DSM the Psychiatric Bible and the Creation Of Mental Disorders. New York: The Free Press. 
Mengal, P. (2000/2001). La constitution de la psychologie comme domaine du savoir aux XVI ${ }^{\text {ème }}$ et XVII İme siècles. Revue d'histoire des sciences humaines 2, 5-27.

Mill, J.S. (1882 [1843]). A System of Logic (8th edition). New York: Harper \& Brothers.

Moncrieff, J. (1995). Lithium revisited. The British Journal of Psychiatry. 167, 569-573.

Moncrieff, J. et Kirsch, I. (2005). Efficacy of antidepressants in adults. British Medical Journal, 331, 155 doi: 10.1136

Moncrieff, J. et Timimi, S. (2010). Is ADHD a valid diagnosis in adults? No. British Medical Journal, 340 : c547 doi: 10.1136/bmj. c547

Panksepp, J. (1998). Affective Neuroscience: The Foundations of Human and Animal Emotions. Oxford: Oxford University Press.

Pourcher, E., (1993). Organic brain dysfunction and cognitive deficits in young schizophrenic patients with tardive dyskinesia. Brain and Cognition 23, 81-87.

Snellius, R. (1594). Partitiones Physicae, Hanoviae, apud Guilielmum Antoninum.

Solms, M. (1997). The Neuropsychology of Dreams: A Clinico-Anatomical Study. Hillsdale: Lawrence Erlbaum.

Solms, M. et Turnbull, O. (2002). The Brain and the Inner World: An Introduction to the Neuroscience of Subjective Experience. New York: Other Press.

Stremler, E. et Castel, P-H. (2009). Pour une histoire des débuts de la neuropsychanalyse: premiers éléments de réflexion à partir de sources inédites. Dans L. Ouss et B. Golse (eds.), Vers une neuropsychanalyse? Paris: Odile Jacob.

Van de Vijver, G. (2010). Het spoor van de psychoanalyse - Een schuinse pas ten aanzien van alle mogelijke aanpassing, Tijdschrift voor Psychoanalyse, 16, 4, 229-239.

Van de Vijver, G. et Demarest, B. (2013). Objectivity: its meaning, its limitations, its fateful objections. Dans G. Olms Verlag, G. Van de Vijver et B. Demarest (eds.), Objectivity after Kant. Its Meaning its Limitations, its Fateful Objections (p. 7-28). Hidesheim, Allemagne: Georg Olm Verlag.

Waddington, J. et al. (1998). Mortality in schizophrenia: Antipsychotic polypharmacy and absence of adjunctive anticholinergics over the course of a 10-year prospective study. British Journal of Psychology, 173, 325-329.

Waddington, J. (1993). Cognitive dysfunction in schizophrenia: organic vulnerability factor or state marker for tardive dyskinesia? Brain and Cognition 23, 56-70.

Wade, J. (1993). Factors related to the severity of tardive dyskinesia. Brain and Cognition 23, 71-80.

Warren, M. et Warren, P. (1968). Helmholtz on Perception: its Physiology and Development. New York: John Wiley \& Sons.

Watters, E. (2010). Crazy Like Us: The Globalization of the American Psyche. New York, NY: Free Press, 306.

Wunderink, L., Nienhuis, F.J., Sytema, S., Slooff, C.J., Knegtering, R. et Wiersma, D. (2007). Guided discontinuation versus maintenance treatment in remitted first-episode psychosis: relapse rates and functional outcome. Journal of Clinical Psychiatry 68, 654-661.

Wundt, W. (1867). Über die Physik der Zelle in ihrer Beziehung zu den allgemeinen Prinzipien der Naturforschung. In: Handbuch der medicinischen Physik. Erlangen: Enke.

Wundt, W. (1874). Grundzüge der physiologischen Psychologie. Leipzig: Engelmann.

Wundt, W. (1882). Die Aufgaben der experimentellen Psychologie. Unsere Zeit 3: 389-406. 